\title{
Analysis and optimization of diode end-pumped solid-state lasers: application to $\mathrm{Nd}^{3+}: \mathrm{YVO}_{4}$ lasers at 1064 and $1342 \mathrm{~nm}$
}

\author{
Alphan Sennaroglu, MEMBER SPIE \\ Adnan Kurt \\ Sandrine Buhours \\ Koç University \\ Department of Physics \\ Laser Research Laboratory \\ Rumelifeneri Yolu, Sariyer, \\ Istanbul 34450 Turkey \\ E-mail: asennar@ku.edu.tr
}

\begin{abstract}
We describe a systematic procedure that uses experimental and numerical methods to analyze the continuous-wave power performance of diode end-pumped solid-state lasers. For the general case, saturation, excited-state absorption, and thermal lensing effects are considered and integral equations are derived to study the evolution of the pump and laser beams in the gain medium. As an application of the method, we consider two different diode end-pumped $\mathrm{Nd}^{3+}: \mathrm{YVO}_{4}$ lasers operating at 1064 and $1342 \mathrm{~nm}$. Experimental efficiency data were first analyzed to determine the stimulated emission cross sections and the resonator losses. The best-fit laser parameters were then used to calculate the optimum crystal length that maximizes the output power of the laser. The described method should prove useful in the design of a wide range of efficient diode-pumped solid-state lasers. (c) 2005 Society of PhotoOptical Instrumentation Engineers. [DOI: 10.1117/1.1915307]
\end{abstract}

Subject terms: solid-state lasers; modeling of solid-state lasers; Nd:vanadate lasers; diode-pumped lasers; thermal effects.

Paper 040398 received Jun. 22, 2004; revised manuscript received Nov. 24, 2004; accepted for publication Dec. 7, 2004; published online May 12, 2005.

\section{Introduction}

Power performance of solid-state lasers depends on the interplay of several factors including the strength of stimulated emission, presence of excited-state absorption, pumpinduced thermal lensing, the influence of nonradiative decay, and pump brightness. Let us consider a continuouswave (cw) diode end-pumped solid-state laser as an example. The choice of the active ion concentration is very critical because departure from the optimum amount can lead to insufficient pump absorption on the one hand or enhanced nonradiative decay on the other. Once the optimum concentration is determined through spectroscopic studies, the crystal length cannot be chosen arbitrarily to obtain a desired amount of absorption. In the case of a three-level system, this is because of the possibility of reabsorption losses at the lasing wavelength. Even if reabsorption is not an issue, as in the case of a four-level laser, the pump beam quality could limit the maximum crystal length that can be used. This is typically the case in medium to high-average power diode end-pumped solid-state lasers where the pump diodes used have low brightness with large $\mathrm{M}^{2}$ values and the Rayleigh range in the gain medium is much shorter than that of the ideal Gaussian beam with the same beam waist. It is therefore important to develop a model that can account for all of the effects mentioned above. Such a model serves two purposes. First, it can be used to determine the values of the important laser parameters such as stimulated emission cross section and passive resonator losses from the experimental laser efficiency data. Second, once the parameters of the laser sys-

0091-3286/2005/\$22.00 @ 2005 SPIE tem are determined, the model can be used to determine the optimum crystal characteristics that maximize the output power.

As an extension of previous studies, ${ }^{1-5}$ we describe a rigorous, systematic procedure for the analysis and optimization of a wide range of $\mathrm{cw}$ diode end-pumped solid-state lasers. First, we develop a model with which the power performance of the laser can be analyzed to determine the stimulated emission cross section and the passive cavity losses. The best-fit values of these parameters are then used to determine the optimum crystal length that maximizes the output power. The model discussed in this paper has two novel features. First, it gives a complete description of pump-laser interactions in solid-state gain media by accounting for most of the important effects such as excitedstate absorption at both the pump and the laser wavelengths, absorption saturation, gain saturation, and pumpinduced thermal lensing. Integral equations that describe the evolution of the pump and the laser powers are then derived for a four-level system. The results can be readily extended to three-level and quasi-three-level systems. Second, the model gives a generalized analysis of resonator stability in the presence of thermal lensing. In particular, the gain medium is treated as a distributed thermal lens and the stability is determined by calculating the maximum pump power up to which a self-consistent Gaussian beam solution can be obtained. As an application of the method, we consider two diode-end-pumped $\mathrm{Nd}^{3+}: \mathrm{YVO}_{4}$ lasers operating at 1064 and $1342 \mathrm{~nm}$. By analyzing the laser efficiency data, the stimulated emission cross sections at 1064 and $1342 \mathrm{~nm}$ were determined to be $7.7 \times 10^{-19} \mathrm{~cm}^{2}$ and $2.2 \times 10^{-19} \mathrm{~cm}^{2}$, respectively. The best-fit data were then used to determine the optimum crystal lengths for which 


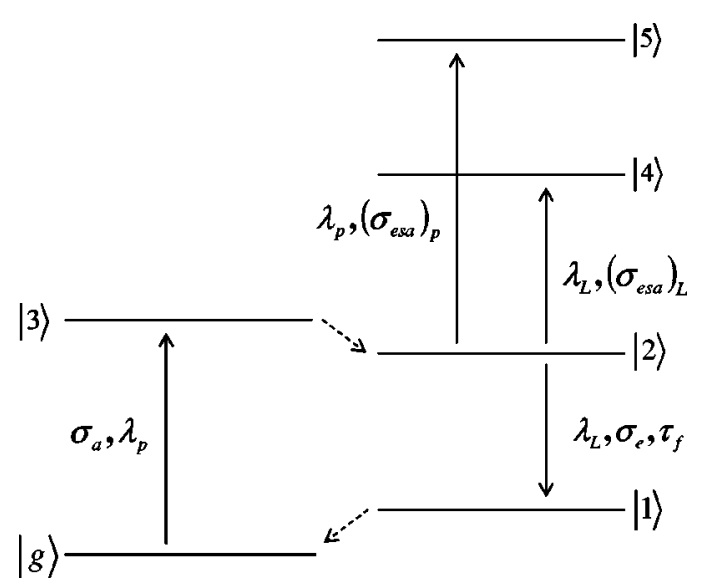

Fig. 1 Modified energy-level diagram of a four-level solid-state gain medium.

the output power is maximized for both resonator configurations.

\section{Description of the Model}

\subsection{Rate Equation Formulation}

We consider a modified four-level system that accurately describes a wide range of solid-state laser media. Excitedstate absorption, saturation, and thermal lensing effects are taken into account. The results obtained here can be easily extended to the case of three-level or quasi-three-level systems as well. Figure 1 shows the energy-level diagram of the system. The population density $N_{2}(r, z)$ of the upper laser level obeys the rate equation

$$
\begin{aligned}
\frac{d N_{2}(r, z)}{d t}= & \frac{\sigma_{a} \lambda_{p} I_{p}(r, z)}{h c} N_{g}(r, z) \\
& -N_{2}(r, z)\left(\frac{\sigma_{e} \lambda_{L} I_{c}(r, z)}{h c}+\frac{1}{\tau_{f}}\right),
\end{aligned}
$$

where $h$ is Planck's constant, $c$ is the speed of light, $N_{g}(r, z)$ is the population density of the ground state $|g\rangle$, $I_{p}(r, z)$ is the intensity of the pump radiation at $\lambda_{p}, I_{c}(r, z)$ is the total cavity intensity $\left[I_{c}(r, z)=I_{L}^{+}(r, z)+I_{L}^{-}(r, z)\right.$, $I_{L}^{ \pm}(r, z)=$ counter propagating intensities inside the laser cavity at $\left.\lambda_{L}\right], \sigma_{a}$ is the absorption cross section at $\lambda_{p}, \sigma_{e}$ is the emission cross section at $\lambda_{L}$, and $\tau_{f}$ is the fluorescence lifetime.

In this analysis, we concentrate on the steady-state solution of Eq. (1). Note that under the assumptions of the model, $N_{2}(r, z)+N_{g}(r, z)=N_{T}$, where $N_{T}$ is the total ion density. The steady-state expression for $N_{2}(r, z)$ can therefore be written in terms of $N_{T}$ and the pump and laser intensities as

$$
N_{2}(r, z)=N_{T} \frac{\frac{I_{p}(r, z)}{I_{s a}}}{1+\frac{I_{c}(r, z)}{I_{s e}}+\frac{I_{p}(r, z)}{I_{s a}}} .
$$

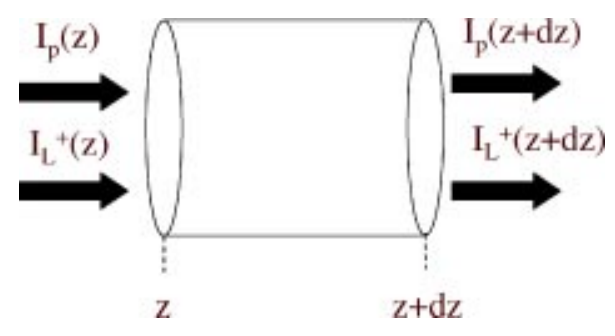

Fig. 2 A section of the gain medium of length $d z$, showing the pump and the forward-propagating laser beams.

Here, $I_{s a}\left(I_{s a}=h c / \sigma_{a} \lambda_{p} \tau_{f}\right)$ and $I_{s e}\left(I_{s e}=h c / \sigma_{e} \lambda_{L} \tau_{f}\right)$ are the absorption and emission saturation intensities, respectively. In order to derive the differential equations satisfied by the pump and the laser powers, we consider a thin slab of the laser-active material as sketched in Fig. 2. The thickness is $d z$. Once the slab is placed inside a resonator and lasing threshold is achieved, there will be two counter propagating laser intensities $I_{L}^{ \pm}(r, z)$ in addition to the pump beam intensity $I_{p}(r, z)$. In Fig. 2 , only $I_{p}$ and $I_{L}^{+}$are shown. The total number of photons removed from the pump beam per unit time will be equal to the number of ions that are excited to higher levels via stimulated groundstate absorption and excited-state absorption. This gives

$\frac{\partial I_{p}}{\partial z}=-\left(\sigma_{a} N_{g}+\left(\sigma_{e s a}\right)_{p} N_{2}\right) I_{p}$,

where $\left(\sigma_{\text {esa }}\right)_{p}$ is the excited-state absorption cross section at the pump wavelength $\lambda_{p}$. The spatial dependence of the intensities was not explicitly shown in Eq. (3) for the sake of clarity. Defining the normalized strength $f_{p}$ of the excited-state absorption at $\lambda_{p}$ as

$f_{p}=\frac{\left(\sigma_{e s a}\right)_{p}}{\sigma_{a}}$

and by using the steady-state expressions for $N_{2}$ and $N_{g}$, the differential equation describing the spatial evolution of the pump intensity can be written as

$\frac{\partial I_{p}}{\partial z}=-\alpha_{p 0} I_{p}\left[\frac{1+\frac{I_{c}}{I_{s e}}+f_{p} \frac{I_{p}}{I_{s a}}}{1+\frac{I_{p}}{I_{s a}}+\frac{I_{c}}{I_{s e}}}\right]$.

In Eq. (5), $\alpha_{p 0}\left(\alpha_{p 0}=N_{T} \sigma_{a}\right)$ is the small-signal differential pump absorption coefficient. In the case of the counterpropagating laser beams, one obtains

$\frac{\partial I_{L}^{ \pm}}{\partial z}= \pm I_{L}^{ \pm}\left[\frac{g_{T}\left(1-f_{L}\right) \frac{I_{p}}{I_{s a}}}{1+\frac{I_{p}}{I_{s a}}+\frac{I_{c}}{I_{s e}}}-\alpha_{L 0}\right]$,

where $f_{L}\left[f_{L}=\left(\sigma_{\text {esa }}\right)_{L} / \sigma_{e},\left(\sigma_{\text {esa }}\right)_{L}=\right.$ excited-state absorption at the laser wavelength $\left.\lambda_{L}\right]$ is the normalized excited- 
state absorption strength at the laser wavelength $\lambda_{L}$ and $g_{T}\left(g_{T}=N_{T} \sigma_{e}\right)$ is the maximum extractable small-signal differential gain coefficient. In addition, $\alpha_{L 0}$ is introduced to account for the distributed losses at the laser wavelength of $\lambda_{L}$.

In order to derive the differential equations satisfied by the beam powers, we assume that the pump $\left(I_{p}\right)$ and the laser $\left[I_{L}^{ \pm}(r, z)\right]$ intensities have a transverse distribution given by

$I_{p}(r, z)=P_{p}(z) \Phi_{p}(r, z)$

$I_{L}^{ \pm}(r, z)=P_{L}^{ \pm}(z) \Phi_{L}(r, z)$.

In Eq. (7), $P_{p}(z)$ and $P_{L}^{ \pm}(z)$ are the pump and laser powers at a specific location $z$. In addition, the normalized transverse distributions $\Phi_{L}$ and $\Phi_{P}$ are assumed to be Gaussian, given by

$\Phi_{L}=\frac{2}{\pi \omega_{L}^{2}} \exp \left(-\frac{2 r^{2}}{\omega_{L}^{2}}\right)$

$\Phi_{P}=\frac{2}{\pi \omega_{p}^{2}} \exp \left(-\frac{2 r^{2}}{\omega_{p}^{2}}\right)$,

where $\omega_{p}$ and $\omega_{L}$ are the respective pump and laser spotsize functions; $\omega_{p}$ and $\omega_{L}$ are functions of $z$. By integrating Eqs. (5) and (6) over the beam cross section and by using Eq. (7), we obtain the following set of differential equations for $P_{p}(z)$ and $P_{L}^{ \pm}(z)$ :

$\frac{d P_{p}}{d z}=-\alpha_{p 0} P_{p} \int_{0}^{\infty} d r 2 \pi r \Phi_{p}\left[\frac{1+\frac{P_{c}}{I_{s e}} \Phi_{L}+f_{p} \frac{P_{p} \Phi_{p}}{I_{s a}}}{1+\frac{P_{p} \Phi_{p}}{I_{s a}}+\frac{P_{c} \Phi_{L}}{I_{s e}}}\right]$

$$
\begin{aligned}
\frac{d P_{L}^{ \pm}}{d z}= \pm P_{L}^{ \pm}\left[g_{T}\left(1-f_{L}\right) P_{p}\right. \\
\\
\left.\times \int_{0}^{\infty} \frac{d r 2 \pi r \frac{\Phi_{L} \Phi_{p}}{I_{s a}}}{1+\frac{P_{p} \Phi_{p}}{I_{s a}}+\frac{P_{c} \Phi_{L}}{I_{s e}}}-\alpha_{L 0}\right] .
\end{aligned}
$$

Note that from Eq. (10), it can be further shown that

$P_{L}^{+} P_{L}^{-}=$constant

at any value of $z$.

\subsection{Thermal Effects}

The unused pump power leads to thermal lensing effects inside the gain medium and can change the spot-size distribution of the cavity beam. In this section, we first derive the expression for the heat source and describe how pump- induced thermal lensing can be taken into account. The spontaneous decay of the ions from $|2\rangle$ to $|1\rangle$ consists of a radiative and a nonradiative component. The overall decay rate $\left(1 / \tau_{f}\right)$ can be expressed as

$\frac{1}{\tau_{f}}=\frac{1}{\tau_{r}}+w_{n r}$,

where $\tau_{r}$ is the radiative lifetime and $w_{n r}$ represents the decay rate due to nonradiative processes. The power $p_{\text {rad }}$ radiated at the emission wavelength of $\lambda_{L}$ per unit volume consists of spontaneous and stimulated terms and is given by

$p_{\text {rad }}=\frac{h c}{\lambda_{L}} \frac{N_{2}}{\tau_{f}}\left[\frac{\tau_{f}}{\tau_{r}}+\frac{I_{c}}{I_{s e}}\right]$.

The pump photons at the wavelength of $\lambda_{p}$ can undergo ground-state or excited-state absorption. The total absorbed power $p_{\text {abs }}$ per unit volume therefore becomes

$p_{a b s}=\frac{h c}{\lambda_{p}} \frac{\sigma_{a} I_{p} \lambda_{p}}{h c}\left(N_{g}+f_{p} N_{2}\right)$.

By using the steady-state expressions for $N_{2}$ and $N_{g}$, the rate $h$ of heat deposition per unit volume can be calculated from

$$
\begin{aligned}
h & =p_{a b s}-p_{\text {rad }} \\
& =\frac{\alpha_{p 0} I_{p}}{1+\frac{I_{p}}{I_{s a}}+\frac{I_{c}}{I_{s e}}}\left[\left(1+\frac{I_{c}}{I_{s e}}+f_{p} \frac{I_{p}}{I_{s a}}\right)-\frac{\lambda_{p}}{\lambda_{L}}\left(\frac{\tau_{f}}{\tau_{r}}+\frac{I_{c}}{I_{s e}}\right)\right] .
\end{aligned}
$$

Various limiting cases of Eq. (15) are of interest. In particular, consider the case where $I_{c}$ is low and pump saturation is negligible. Then $h$ can be approximated as

$$
h=\alpha_{p 0} I_{p}\left(1-\frac{\lambda_{p}}{\lambda_{L}} \frac{\tau_{f}}{\tau_{r}}\right) .
$$

Also note that for very large values of $I_{c}, h$ approaches the quantum defect limit of

$h=\alpha_{p 0} I_{p}\left(1-\frac{\lambda_{p}}{\lambda_{L}}\right)$.

The heat due to the unused pump causes thermal lensing. The temperature distribution $T(r, z)$ inside the laser rod may be approximated as

$T(r, z)=T_{1}(z)-T_{2}(z) r^{2}$,

where the quadratic temperature coefficient $T_{2}(z)$ can be calculated in terms of the axial value $h_{0}(r=0)$ of the heat source from 
$T_{2}(z)=\frac{h_{0}(z)}{4 \kappa}$.

Here, $\kappa$ is the heat conductivity of the host crystal. ${ }^{6}$ To first order, change in the local value of the refractive index will be proportional to the temperature rise according to

$n(r, z)=n_{0}+n_{T}\left(T(r, z)-T_{r}\right)$.

In Eq. (20), $n_{0}$ is the refractive index at a reference temperature $T_{r}$ and $n_{T}=d n / d T$ is the thermal index coefficient. Hence, the refractive index of the crystal will vary according to

$n(r, z)=n_{0}\left(1+\frac{n_{T}}{n_{0}}\left(T_{1}(z)-T_{r}\right)-\frac{\beta(z)^{2} r^{2}}{2}\right)$,

where the quadratic index coefficient $\beta(z)$ is given by

$\beta(z)=\sqrt{\frac{2 n_{T} T_{2}(z)}{n_{0}}}$.

For typical values of $n_{T}$ and $n_{0}$, the axial variation $\left(n_{T} / n_{0}\right)\left(T_{1}(z)-T_{r}\right)$ in $n(r, z)$ may be neglected. The variation of the Gaussian beam parameter $q(z)$ due to thermal lensing effects can be calculated by solving the differential equation

$\frac{1}{q(z)}+\frac{d}{d z}\left(\frac{1}{q(z)}\right)+\beta(z)^{2}=0$.

\section{Modeling of the Diode End-Pumped Nd: $\mathrm{YVO}_{4}$ Laser}

In our study, we investigated the continuous-wave power performance of two diode end-pumped $\mathrm{Nd}: \mathrm{YVO}_{4}$ lasers operating at 1064 and $1342 \mathrm{~nm}$. In this particular case, a number of simplifying assumptions may be made in the general model that was discussed in Section 2. The samples used in our study had 0.5 at. \% Nd. At this doping level, the

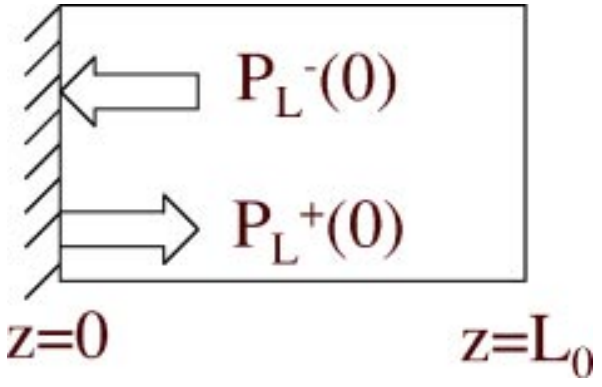

Fig. 3 The input face of the gain crystal with the forward- and backward-propagating laser beams. The gain crystal extends from $z=0$ to $z=L_{0}$.

nonradiative decay rate $\left[w_{n r}\right.$ in Eq. (12)] of the excited ions is negligible so we assumed that $\tau_{f} \approx \tau_{r} .{ }^{7}$ Furthermore, excited-state absorption at the pump and laser wavelengths and saturation of the pump were assumed to be negligible. This gives $f_{L}=0, f_{p}=0$, and $I_{s a}=\infty$ in Eqs. (5) and (6). In this limit, $P_{p}(z)$ is decoupled from $P_{L}^{ \pm}(z)$ and has the wellknown exponential decay solution:

$P_{p}(z)=P_{p i} \exp \left(-\alpha_{p 0} z\right)$.

In Eq. (24), $P_{p i}$ is the incident pump power at the front face $(z=0)$ of the gain medium. In the experimental configurations used in our study, the entrance face of the crystal was coated to be highly reflecting at the lasing wavelength, as shown in Fig. 3. This gives $P_{L}^{+}(z=0)=P_{L}^{-}(z=0)=P_{L i}$ where, $P_{L i}$ is the intracavity laser power at $z=0$. At an arbitrary value of $z$, the power $P_{L}^{-}(z)$ of the backwardpropagating laser beam can therefore be expressed in terms of $P_{L}^{+}(z)$ as [see Eq. (11)]

$P_{L}^{-}(z)=\frac{P_{L i}^{2}}{P_{L}^{+}(z)}$

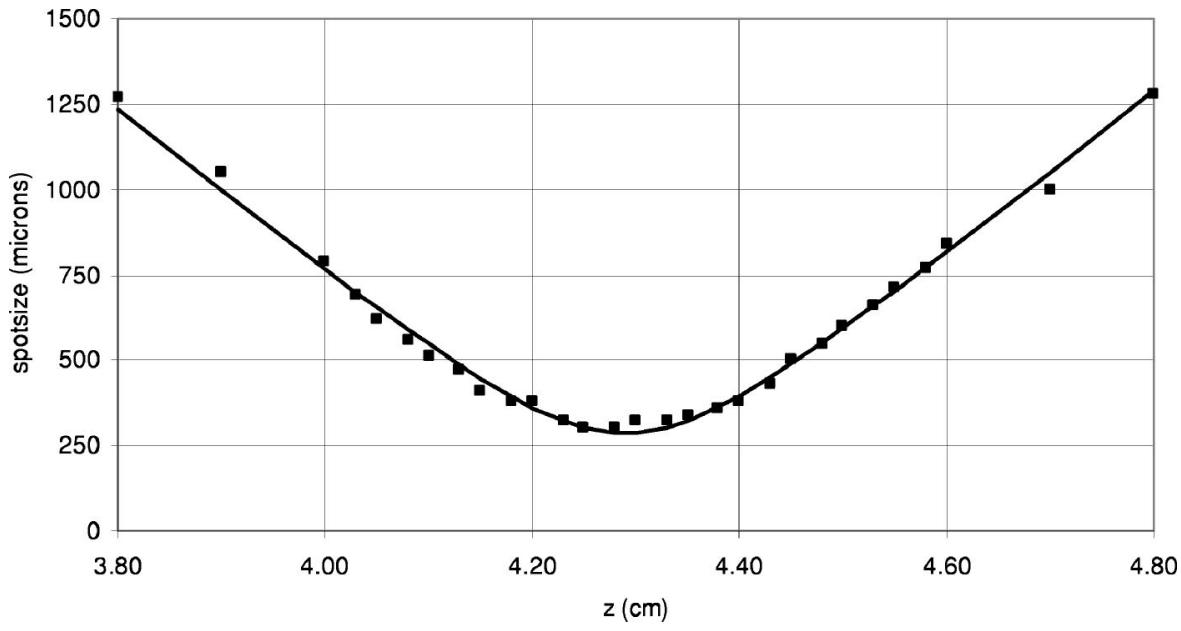

Fig. 4 Measured variation of the pump spot-size function as a function of relative position. The pump beam waist and the $\mathrm{M}^{2}$ factor were determined to be $287 \mu \mathrm{m}$ and 275 . 


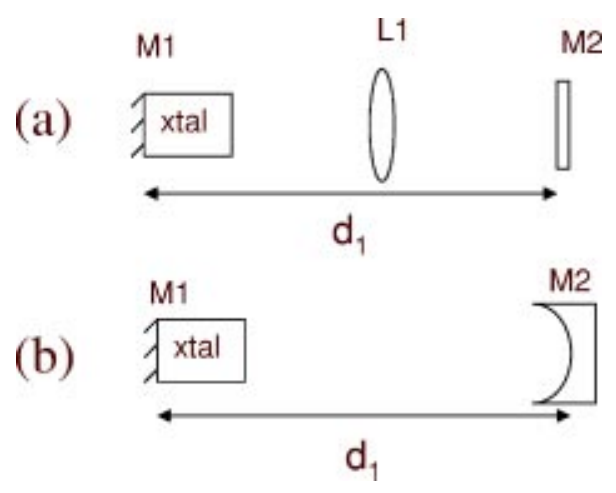

Fig. 5 Schematics of the diode end-pumped $\mathrm{Nd}: \mathrm{YVO}_{4}$ laser setups operated at (a) $1064 \mathrm{~nm}$ and (b) $1342 \mathrm{~nm}$.

By using Eqs. (24) and (25), the evolution equations [Eqs. (9) and (10)] for the pump and laser powers are hence reduced to a single equation for $P_{L}^{+}(z)$ inside the gain medium. For a specific resonator geometry and incident pump power, we determine the intracavity power $P_{L i}$ for which the net round-trip gain of $P_{L}^{+}(z)$ is unity. If the losses of the cavity elements between the exit face of the crystal and the output coupler are negligible, then the output power $P_{\text {out }}$ of the resonator is determined from

$P_{\text {out }}=P_{L}^{+}\left(z=L_{0}\right) T$,

where $P_{L}^{+}\left(z=L_{0}\right)$ is the forward intracavity power at the exit face of the crystal (see Fig. 3), $L_{0}$ is the crystal length, and $T$ is the transmission of the output coupler. Finally, the threshold pump power $P_{\text {thres }}$ required for lasing is determined by solving the round-trip equation for $P_{L}^{+}(z)$ in the limit of vanishing $P_{L i}$.

The effect of thermal lensing is to modify the cavity spot-size distribution and hence the transverse mode distribution $\Phi_{L}$ that appears in the power equations. By using Eq. (16) and the simplifying assumptions outlined above, the expression for the axial heat source $h_{0}$ can be expressed in the low- $I_{c}$ limit as

$h_{0}=h(r=0, z)=\frac{2 \alpha_{p 0} P_{i}}{\pi \omega_{p}^{2}(z)} \exp \left(-\alpha_{p 0} z\right)\left(1-\frac{\lambda_{p}}{\lambda_{L}}\right)$.

The pump spot-size function $\omega_{p}(z)$ was assumed to have the functional form

$\omega_{p}(z)=\omega_{p 0} \sqrt{1+\left(\frac{z-z_{f p}}{z_{R p}}\right)^{2}}$,

where $\omega_{p 0}$ is the pump beam waist, $z_{f p}$ is the beam waist location, and $z_{R p}\left(z_{R f}=n_{0} \pi \omega_{p 0}^{2} / M^{2} \lambda_{p}, n_{0}\right.$ is the refractive index of the crystal, and $M^{2}$ is the beam quality factor) is the Rayleigh range of the pump. The $\mathrm{M}^{2}$ factor and the beam waist $\omega_{p 0}$ need to be determined from the experimentally measured spot-size data. In our experiments, the spotsize function $\omega_{p}(z)$ was measured by using the knife-edge technique. The measured variation of $\omega_{p}(z)$ as a function

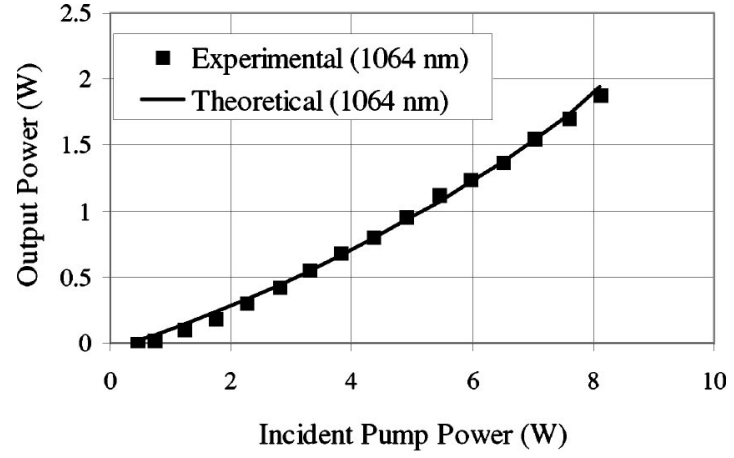

(a)

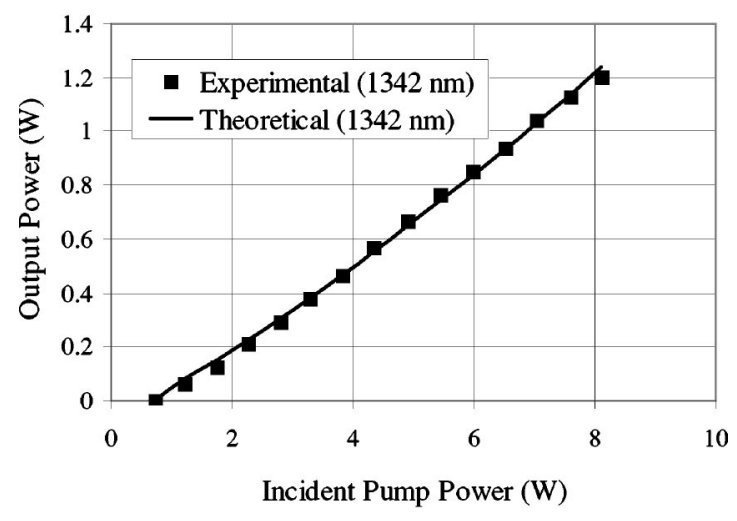

(b)

Fig. 6 Measured and calculated variation of the output power as a function of the incident pump power for the $\mathrm{Nd}: \mathrm{YVO}_{4}$ laser operated at (a) $1064 \mathrm{~nm}$ and (b) $1342 \mathrm{~nm}$.

of relative position is displayed in Fig. 4. The best-fit values of the beam waist and $\mathrm{M}^{2}$ factor were found to be 287 $\mu \mathrm{m}$ and 275 , respectively.

In the analysis of thermal lensing, we assumed that the cavity spot-size distribution has a Gaussian transverse profile and used Eq. (23) to determine how the q-parameter of the beam is modified inside the gain medium. The effect of the other cavity elements on the propagation of the beam was analyzed by using standard matrix methods. Note that the effect of thermal lensing inside the gain medium can no longer be expressed as a $2 \times 2$ matrix. In this case, the effect of the gain medium and the remaining optical elements were separately treated. The self-consistent q-parameter $q_{\mathrm{sc}}(z)$ of the cavity under thermal loading is determined by solving the equation

$q_{s c}^{n+1}(z)=q_{s c}^{n}(z)$,

where the superscript $n$ denotes the q-parameter after $n$ round-trips. The cavity spot-size function $\omega_{L}(z)$ is determined from $q_{\mathrm{sc}}(z)$ and used in the calculation of the output power. Equation (29) can also be used to investigate the role of thermal lensing on cavity stability. Since the effect of one round-trip on the q-parameter can no longer be written as a $2 \times 2$ matrix, we used a modified stability criterion. We observed that for a fixed cavity configuration, selfconsistent solution of Eq. (29) can be obtained up a maximum incident pump power $P_{\text {stab }}$. So, for a given configu- 


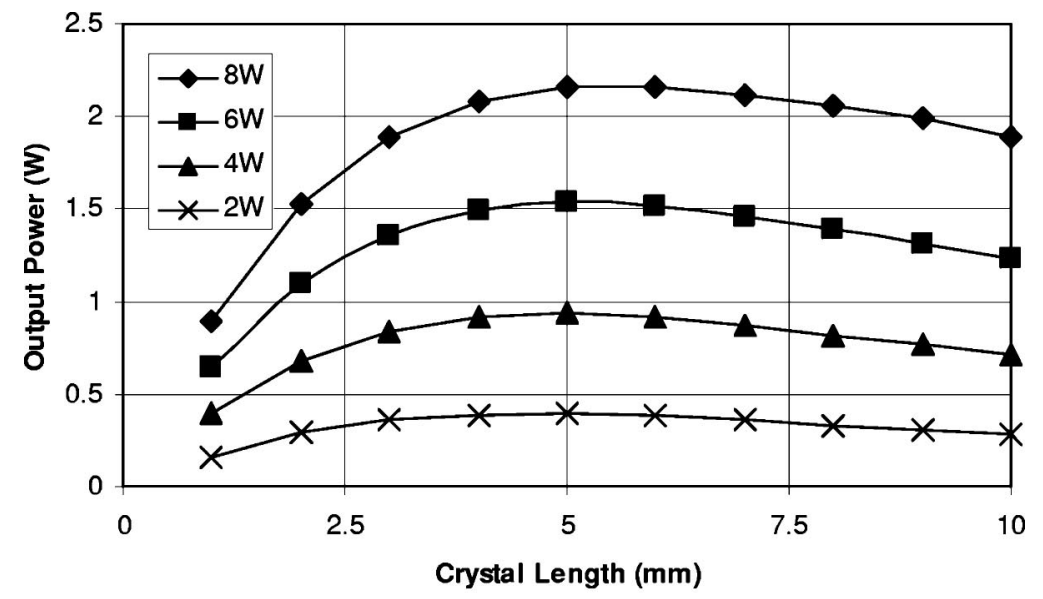

(a)

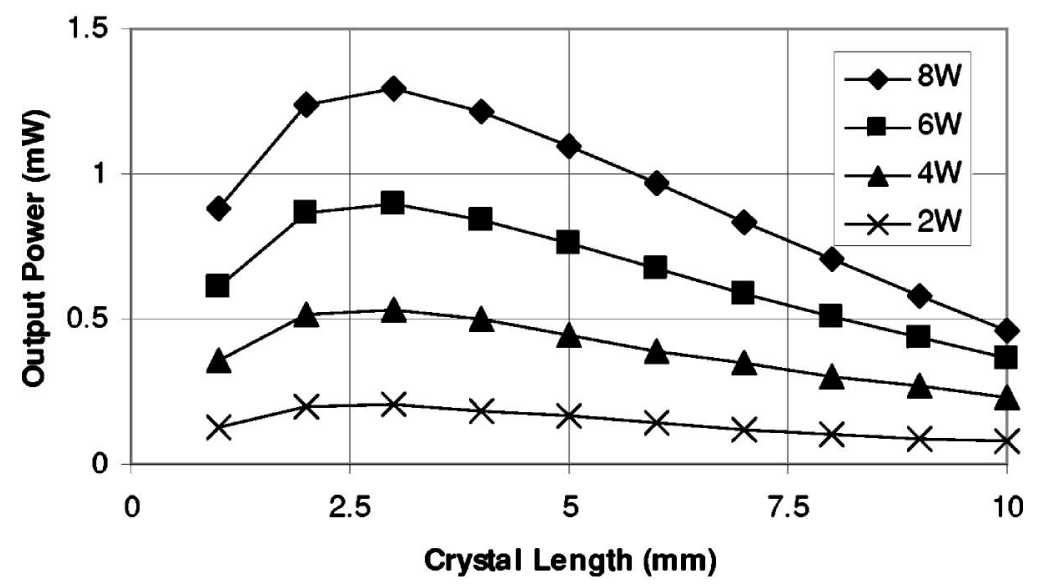

(b)

Fig. 7 Calculated variation of the output power as a function of the crystal length at (a) $1064 \mathrm{~nm}$ and (b) $1342 \mathrm{~nm}$ for incident pump powers of 2, 4, 6, and $8 \mathrm{~W}$.

ration, $P_{\text {stab }}$ was first determined and spot-size calculations were performed only when the incident pump power $P_{p i}$ $<P_{\text {stab }}$. So, the inequality $P_{p i} \leqslant P_{\text {stab }}$ replaces the more commonly known stability condition $|A+D| / 2 \leqslant 1$ in our case.

\section{Results}

The experimental arrangements of the $\mathrm{Nd}: \mathrm{YVO}_{4}$ lasers operated at 1064 and $1342 \mathrm{~nm}$ are shown in Figs. 5(a) and 5 (b), respectively. In each case, normal-cut $\mathrm{Nd}: \mathrm{YVO}_{4}$ crystals with 0.5 at. $\% \mathrm{Nd}$ were used. The front end of each crystal, which was coated to transmit the pump and reflect the laser beam, also served as the input mirror (M1). The second crystal surface was antireflection-coated at the lasing wavelength. Both resonators were end-pumped by a fiber-coupled diode laser operating at $804 \mathrm{~nm}$. In the 1064-nm setup shown in Fig. 5(a), a 10-mm-long gain crystal was used and the resonator contained an antireflectioncoated intracavity lens (L1, focal length $=10 \mathrm{~cm}$ ) between M1 and the flat output coupler $[\mathrm{M} 2$ in Fig. 5(a), transmission $=2.1 \%$ at $1064 \mathrm{~nm}]$. The total cavity length $d_{1}$ was $15.2 \mathrm{~cm}$. In the case of the 1342-nm setup, the gain medium was $4 \mathrm{~mm}$ long and the cavity was completed with a curved $2 \%$ transmitting output coupler [M2 in Fig. 5(b), radius $=10 \mathrm{~cm}]$. Here, the total cavity length $d_{1}$ was $6 \mathrm{~cm}$.

In the analysis, small-signal loss coefficient $\left(\alpha_{L 0}\right)$ and the stimulated emission cross section $\left(\sigma_{e}\right)$ were used as adjustable parameters to obtain the best fit between the calculated and measured efficiency data. For a given cavity configuration, the pump beam waist $\left(z_{f p}\right)$ was first varied to minimize the threshold pump power. Figures 6(a) and 6(b) show the calculated and measured variation of the output power as a function of the incident pump power at 1064 and $1342 \mathrm{~nm}$, respectively. The best fit between experimental data and theoretical model was obtained for $\alpha_{L 0}$ $=0.015 \mathrm{~cm}^{-1}$ (corresponding to a round-trip cavity loss of $3 \%$ ) and $\sigma_{e}=7.7 \times 10^{-19} \mathrm{~cm}^{2}$. The emission cross-section value determined in our analysis is close to that reported in Ref. 8. In the case of the $1342-\mathrm{nm}$ resonator, the best-fit values of $\alpha_{L 0}$ and $\sigma_{e}$ were determined to be $0.024 \mathrm{~cm}^{-1}$ and $2.2 \times 10^{-19} \mathrm{~cm}^{2}$, respectively.

By using the best-fit values of the small-signal loss coefficient and the stimulated emission cross section, numerical optimization studies were then carried out to determine the crystal length that maximizes the output power. The same resonator configurations shown in Fig. 5 and the same 
pumping configuration were assumed. At each value of the crystal length, the pump beam waist location was first optimized inside the crystal to yield the lowest calculated threshold pump power. Optimization calculations were performed for incident pump powers of $2,4,6$, and $8 \mathrm{~W}$. The results are shown in Figs. 7(a) and 7(b). In the case of the 1064-nm resonator, the optimum crystal length varies from 5 to $6 \mathrm{~mm}$ as the incident pump power is increased from 2 to $8 \mathrm{~W}$. In the case of the 1342-nm resonator, the optimum crystal length remains around $3 \mathrm{~mm}$ for incident pump powers in the $2-8 \mathrm{~W}$ range. We note that optimization studies can also be carried out as a function of other parameters such as crystal absorption. However, in this case, additional data showing the concentration dependence of lifetime are needed since fluorescence quenching effects will be enhanced as the concentration is increased.

\section{Conclusions}

In conclusion, we described a systematic procedure for the analysis and optimization of a wide range of cw diode endpumped solid-state lasers. The model considers a modified four-level system and accounts for excited-state absorption at the pump and the laser wavelengths, gain saturation, and absorption saturation. Integral equations describing the evolution of the pump and the laser powers were derived. Pump-induced thermal lensing was also taken into account. The model was then used to analyze the power performance of two diode end-pumped $\mathrm{Nd}^{3+}$ : $\mathrm{YVO}_{4}$ lasers operating at 1064 and $1342 \mathrm{~nm}$. The stimulated emission cross sections were determined to be $7.7 \times 10^{-19} \mathrm{~cm}^{2}$ and 2.2 $\times 10^{-19} \mathrm{~cm}^{2}$ at 1064 and $1342 \mathrm{~nm}$, respectively. The bestfit values of the laser parameters were then used to determine the optimum crystal lengths that maximize the output power. The dependence of the optimum crystal length on the incident pump power was also investigated. The model that can be readily extended to include other effects should prove useful in the design of efficient diode end-pumped solid-state lasers.

\section{Acknowledgments}

A. Sennaroglu acknowledges the support of the Turkish Academy of Sciences in the framework of the Young Scientist Award Program AS/TUBA-GEBIP/2001-1-11.

\section{References}

1. T. Y. Fan and R. L. Byer, "Modeling and cw operation of a quasithree-level 946 nm Nd:YAG laser," IEEE J. Quantum Electron. 23 605-612 (1987)

2. A. J. Alfrey, "Modeling of longitudinally pumped cw Ti: sapphire laser oscillators," IEEE J. Quantum Electron. 25, 760-766 (1989).

3. P. F. Moulton, "An investigation of the Co: $\mathrm{MgF}_{2}$ laser system," IEEE J. Quantum Electron. 21, 1582-1595 (1985).

4. W. P. Risk, "Modeling of longitudinally pumped solid-state lasers exhibiting reabsorption losses," J. Opt. Soc. Am. B 5, 1412-1423 (1988).
5. A. D. Lieto, P. Minguzzi, A. Pirastu, and V. Magni, "High-power diffraction-limited $\mathrm{Nd}: \mathrm{YVO}_{4}$ continuous-wave lasers at $1.34 \mu \mathrm{m}$," IEEE J. Quantum Electron. 39, 903-909 (2003).

6. A. Sennaroglu, "Continuous-wave thermal loading in saturable absorbers: theory and experiment," Appl. Opt. 36, 9528-9535 (1997).

7. Y. F. Chen, L. J. Lee, T. M. Huang, and C. L. Wang, "Study of high-power diode-end-pumped $\mathrm{Nd}: \mathrm{YVO}_{4}$ laser at $1.34 \mathrm{~mm}$ : influence of Auger upconversion," Opt. Commun. 163, 198-202 (1999).

8. N. Mermilliod, R. Romero, I. Chartier, C. Garapon, and R. Moncorge, "Performance of various diode-pumped $\mathrm{Nd}$ : laser materials. Influence of inhomogeneous broadening," IEEE J. Quantum Electron. 28, 1179-1187 (1992)

Alphan Sennaroglu is a professor in the departments of physics and electrical-electronics engineering. He received his BS, MS, and $\mathrm{PhD}$ degrees in electrical engineering from Cornell University in 1988, 1990, and 1994, respectively. After joining Koç University in 1994, he established the Laser Research Laboratory. Currently, he is also affiliated with the Micro-Nano Technology Center at Koç University. His research interests include solid-state lasers, ultrafast optics, spectroscopy of novel laser and amplifier media, and nonlinear optics. He was a visiting researcher at the Massachusetts Institute of Technology during the 2002-2003 academic year. He served as the program chair for the Solid-State Lasers and Amplifiers Conference during the Photonics Europe 2004 meeting in Strasbourg, France. $\mathrm{He}$ is a senior member of IEEE (Institute of Electrical and Electronics Engineers). He is also a member of OSA (Optical Society of America), SPIE (The International Society for Optical Engineering), Optics Committee of Turkey, Tau Beta Pi, and Eta Kappa Nu. In 1999, he founded the Leos Turkish Chapter of the IEEE Lasers and Electro-Optics Society and served as the chapter president between 1999 and 2003. He received the 2002 International Center for Theoretical Physics/International Commission for Optics Award), 2001 Werner-von-Siemens Award (Koç University), 2001 Tuba (Turkish Academy of Sciences) Young Scientist Award, 1998 Tubitak (Scientific and Technical Research Council of Turkey) Young Scientist Award, Sage Graduate Fellowship (Cornell University, 19891990), Sibley Prize of Electrical Engineering (Cornell University, 1988), and Amideast undergraduate scholarship (1984-1988).

Adnan Kurt received his BS (electrical engineering) and MS (physics) degrees from Boğaziçi University, Istanbul, Turkey, in 1984 and 1987, respectively. Between 1985 and 1990, he was a research assistant in the departments of physics and psychology at Bogazici University, and worked on laser speckle, pulsed gas laser design, neuro-computing, and animal learning. Later, he joined the research group at the Center for Electroneurophysiology at Istanbul University Medical School, and continued work in neurophysiology and human brain studies (1990-1995). He also worked for Mitra Inc. and Teknofil Ltd. doing business in printing, imaging, electrophotography, and instrumentation control. In 1999, he joined the Department of Physics at Koç University as a research engineer. His current research interests include solid-state laser design, optical and electronic instrumentation, and laser spectroscopy.

Sandrine Buhours received her BS and MS degrees in physics and chemistry from Institut National des Sciences Appliquees of Toulouse in 1993 and 2000, respectively. She was a visiting student in the Laser Research Laboratory at Koç University in 2002. Her research interests include solid-state lasers, numerical modeling, and instrumentation. 